EPJ Web of Conferences 49, 15012 (2013)

DOI: $10.1051 /$ epjconf/20134915012

(C) Owned by the authors, published by EDP Sciences, 2013

\title{
Searches for Top and Bottom Squarks in pp Collisions at $\sqrt{s}=8 \mathrm{TeV}$
}

\author{
Benjamin Hooberman ${ }^{1, a}$, for the CMS Collaboration \\ ${ }^{1}$ Fermi National Accelerator Laboratory
}

\begin{abstract}
Supersymmetry is a popular extension to the standard model, which may solve the hierarchy problem without fine-tuning if it introduces top and bottom squarks with masses not larger than several hundred GeV. This note describes three searches for the direct pair production of these particles, based on a sample of $\mathrm{pp}$ collisions data corresponding to approximately $10 \mathrm{fb}^{-1}$ collected by the Compact Muon Solenoid detector at the LHC, at a center-of-mass energy $\sqrt{s}=8 \mathrm{TeV}$. The searches are performed in the single lepton final state focusing on events with large transverse mass, the same-sign dilepton final state, and the all-hadronic final state using the $\alpha_{T}$ quantity. No evidence for the production of top or bottom squarks is observed. The results are used to place stringent constraints on the masses of these particles.
\end{abstract}

\section{Introduction}

Supersymmetry (SUSY) is a popular extension to the standard model (SM) of particle physics, which may explain the 16 orders of magnitude difference between the electroweak and Planck scales (hierarchy problem), introduce a natural candidate for the dark matter weakly-interacting massive particle (WIMP), and lead to the unification of gauge couplings. In order to solve the hierarchy problem naturally (without fine-tuning), SUSY must introduce light top and bottom squarks, the SUSY partners of the top and bottom quarks [1]. If R-parity is conserved, the lightest SUSY particle (LSP), usually the lightest neutralino $\tilde{\chi}_{1}^{0}$, is a stable WIMP. If produced in pp collisions this particle carries away undetected energy and leads to large missing transverse energy $\left(\mathrm{E}_{\mathrm{T}}^{\mathrm{miss}}\right)$. In this note we present the results of searches for top and bottom squarks in final states with $\mathrm{E}_{\mathrm{T}}^{\mathrm{miss}}$, using data collected by the Compact Muon Solenoid (CMS) [2] detector at the Large Hadron Collider (LHC). The data was collected at a center-of-mass energy $\sqrt{s}=8 \mathrm{TeV}$ in 2012 and corresponds to an integrated luminosity of approximately $10 \mathrm{fb}^{-1}$.

Top and bottom squarks may either be produced directly in pairs (direct squark pair production) or in the decays of heavier SUSY particles such as the gluino (gluinomediated squark production). In this note we focus on searches for direct squark pair production; examples of such processes are indicated in Fig. 1. These scenarios may lead to excesses above the SM background expectations in several final states, depending on the mass hierarchy of the SUSY particles. Here we report the results from three searches which focus on the single lepton final state using the transverse mass [3], the same-sign dilepton final state [4], and the all-hadronic final state using the $\alpha_{T}$ quantity [5].

\footnotetext{
ae-mail: benhoob@fnal.gov
}

\section{Search for Top Squark Pair Production in the Single Lepton Final State}

This section presents the results of a dedicated search for the direct pair production of top squarks, based on an integrated luminosity of $9.7 \mathrm{fb}^{-1}$. The decay of the top squark depends on the difference between its mass and that of the $\tilde{\chi}_{1}^{0} \mathrm{LSP}, \Delta m=m_{\tilde{t}}-m_{\tilde{\chi}_{1}^{0}}$. If $\Delta m>m_{t}$, the decay $\tilde{t} \rightarrow t \tilde{\chi}_{1}^{0}$ is expected to have a large branching fraction. If there is a light chargino $\tilde{\chi}_{1}^{ \pm}$, the decay $\tilde{t} \rightarrow b \tilde{\chi}_{1}^{+} \rightarrow b W \tilde{\chi}_{1}^{0}$ may also be significant. The pair production of top squarks decaying to either of these channels leads to events with two b-jets, two W bosons, and two LSPs. Our signal thus resembles SM $t \bar{t}$ production but with larger $\mathrm{E}_{\mathrm{T}}^{\mathrm{miss}}$ from the invisible LSPs. We focus here on the single lepton final state, which has a significant branching fraction due to the presence of two $\mathrm{W}$ bosons in the final state, and smaller SM backgrounds than the all-hadronic final state.

We require the presence of exactly one well-identified and isolated electron (e) or muon $(\mu)$ with transverse momentum $p_{T}>30 \mathrm{GeV}$. We select events with at least four jets with $p_{T}>30 \mathrm{GeV}$, which must be well-separated from the selected leptons. At least one of these jets is required to be consistent with coming from the decay of a heavy flavor hadron, as identified by the Combined Secondary Vertex medium working point (CSVM) b-tagging algorithm [6]. The jet requirements suppress SM backgrounds from W bosons produced in association with jets from initial state radiation (ISR), referred to as the $\mathrm{W}+$ jets background. The $\mathrm{E}_{\mathrm{T}}^{\mathrm{miss}}$ is required to exceed $50 \mathrm{GeV}$, suppressing the background from QCD multijet production.

The SM background satisfying the above requirements is dominated by $t \bar{t}$ production where one $\mathrm{W}$ boson decays hadronically and the other leptonically $(t \bar{t} \rightarrow \ell+$ jets), or where both $\mathrm{W}$ bosons decay leptonically $(t \bar{t} \rightarrow \ell \ell)$. There is a small contribution from $\mathrm{W}+$ jets, as well as a variety 


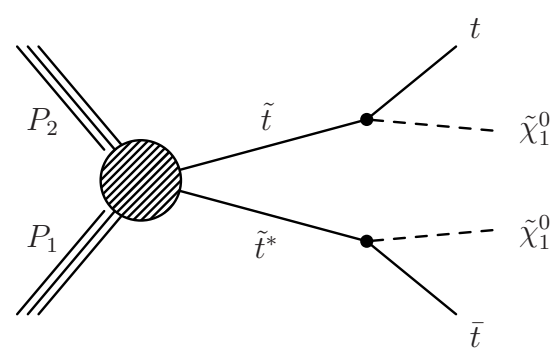

(a)

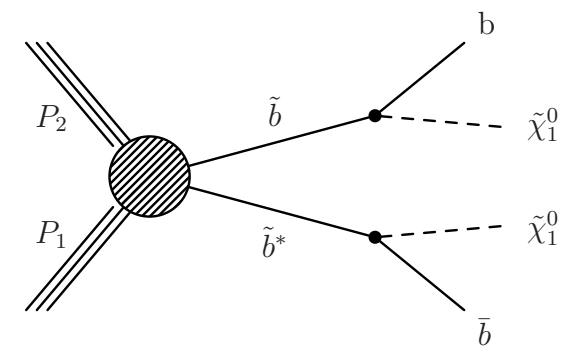

(c)

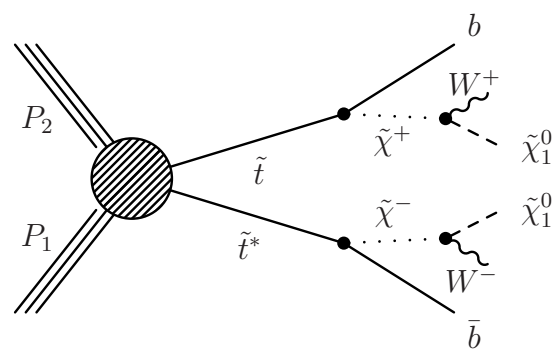

(b)

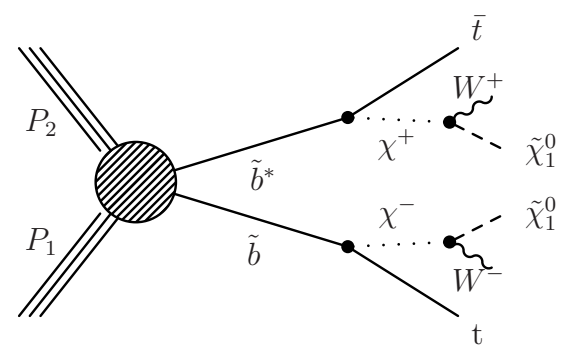

(d)

Figure 1: Example topologies with direct pair production of top (a,b) and bottom (c,d) squarks.

of rare SM processes, dominated by $t \bar{t}$ produced in association with a vector boson $(t \bar{t} W$ and $t \bar{t} Z)$.

To define signal regions, we require the events to have large transverse mass, defined as:

$$
M_{T}=\sqrt{2 p_{T}^{\ell} \mathrm{E}_{\mathrm{T}}^{\mathrm{miss}}(1-\cos (\Delta \phi))},
$$

where $p_{T}^{\ell}$ is the lepton transverse momentum and $\Delta \phi$ is the difference in azimuthal angles between the lepton and $\mathrm{E}_{\mathrm{T}}^{\mathrm{miss}}$. This requirement strongly suppresses the background from $t \bar{t} \rightarrow \ell+$ jets and $\mathrm{W}+$ jets, which have a kinematic endpoint at $M_{T} \approx M_{W}$ since the lepton and neutrino (which produces the $\mathrm{E}_{\mathrm{T}}^{\mathrm{miss}}$ ) are produced together in the decay of the W. For signal events, as well as for the $t \bar{t} \rightarrow \ell \ell$ background, the presence of more than one invisible particle in the final state leads to events with $M_{T}>>M_{W}$. In addition to the $M_{T}$ requirement, we make several $\mathrm{E}_{\mathrm{T}}^{\mathrm{miss}}$ requirements to achieve sensitivity to signals with different mass spectra. Signal regions with large (small) $E_{\mathrm{T}}^{\text {miss }}$ requirements are more sensitive to signals with large (small) values of $\Delta m$.

The dominant background in our signal regions is $t \bar{t} \rightarrow \ell \ell$, which may produce events with large $\mathrm{E}_{\mathrm{T}}^{\mathrm{miss}}$ and $M_{T}$ due to the presence of more than one invisible neutrino. In order for $t \bar{t} \rightarrow \ell \ell$ events to pass the signal region selection, one of the two $\mathrm{W}$ leptons must not be identified, which occurs if it is outside the acceptance, is a hadronic $\tau$ decaying to three charged particles (3-prong decay), is a hadronic $\tau$ decaying to a single charged particle (1-prong decay), or is an electron or muon that fails the lepton identification requirements. The latter two categories are suppressed by vetoing events that contain, in addition to the selected lepton, a charged particle with $p_{T}>10 \mathrm{GeV}$ that is isolated in space from other energetic charged particles. Furthermore, additional jets from initial state or final state radiation (ISR/FSR) are required to satisfy the jet multiplicity requirement $n_{\text {jets }} \geq 4$. To validate and correct the MC modeling of jets from radiation, the MC is compared to data in a dilepton control region dominated by $t \bar{t} \rightarrow \ell \ell$. The MC distribution of $n_{\text {jets }}$ is reweighted to match the corresponding data distribution, resulting in small corrections of a few $\%$.

The SM backgrounds are estimated from events simulated with Monte Carlo (MC) techniques, which are validated and (where necessary) corrected using comparisons to data in control regions. The MC expectation is normalized to data in the $M_{T}$ peak region, in order to remove systematic uncertainties from integrated luminosity and $t \bar{t}$ cross section, and then extrapolated to the large $M_{T}$ region. Correction factors and corresponding systematic uncertainties on the MC extrapolation factors are evaluated by comparing $\mathrm{MC}$ to data in dedicated control regions dominated by $\mathrm{W}+$ jets (obtained by vetoing events with b-jets), $t \bar{t} \rightarrow \ell \ell$ (obtained by requiring two selected leptons), and a mixture of $t \bar{t} \rightarrow \ell \ell$ and $t \bar{t} \rightarrow \ell+$ jets (obtained by requiring a selected lepton and an isolated track). The dominant systematic uncertainty in the background prediction is due to the limited statistical precision in the data control samples used for these tests.

The results of the search are summarized in Table 1, which displays the SM background expectations and the observed data yields in the signal regions. The distribution of $\mathrm{E}_{\mathrm{T}}^{\mathrm{miss}}$ after the requirement $M_{T}>120 \mathrm{GeV}$ is displayed in Fig. 2. Good agreement between the data and the expected background is observed. We find no evidence for the pair production of top squarks.

To interpret the results of our search, we consider top squark pair production where both top squarks decay according to $\tilde{t} \rightarrow t \tilde{\chi}_{1}^{0}$, in Fig. 3 . The model is parameterized by the masses of the top squark and $\tilde{\chi}_{1}^{0}$. We place up- 
Table 1: Summary of results of the search for top squark pairs. For each signal region (SR) the individual background contributions, total background, and observed data yields are indicated. Single lepton top refers to the sum of $t \bar{t} \rightarrow \ell+$ jets and single top production. The uncertainty includes both the statistical and systematic components.

\begin{tabular}{l||c|c|c|c|c|c|c}
\hline Sample & SRA & SRB & SRC & SRD & SRE & SRF & SRG \\
\hline \hline$t \bar{t} \rightarrow \ell \ell$ & $579 \pm 38$ & $328 \pm 37$ & $111 \pm 18$ & $39 \pm 10$ & $14 \pm 6$ & $6.2 \pm 2.9$ & $3.5 \pm 1.8$ \\
Single lepton top & $256 \pm 131$ & $120 \pm 51$ & $29.0 \pm 16.4$ & $7.7 \pm 5.1$ & $3.1 \pm 2.7$ & $1.7 \pm 1.6$ & $0.8 \pm 1.0$ \\
W + jets & $33.5 \pm 8.2$ & $17.5 \pm 4.5$ & $5.54 \pm 1.98$ & $2.0 \pm 1.0$ & $1.0 \pm 0.7$ & $0.7 \pm 0.6$ & $0.3 \pm 0.4$ \\
Rare & $59.0 \pm 29.5$ & $38.5 \pm 19.3$ & $16.1 \pm 8.1$ & $7.7 \pm 3.9$ & $3.6 \pm 1.8$ & $1.5 \pm 0.8$ & $1.1 \pm 0.6$ \\
\hline Total background & $927 \pm 138$ & $504 \pm 65$ & $161 \pm 26$ & $56 \pm 12$ & $22 \pm 7$ & $10 \pm 3$ & $5.7 \pm 2.2$ \\
\hline \hline Data & 861 & 456 & 150 & 61 & 23 & 9 & 3 \\
\hline
\end{tabular}

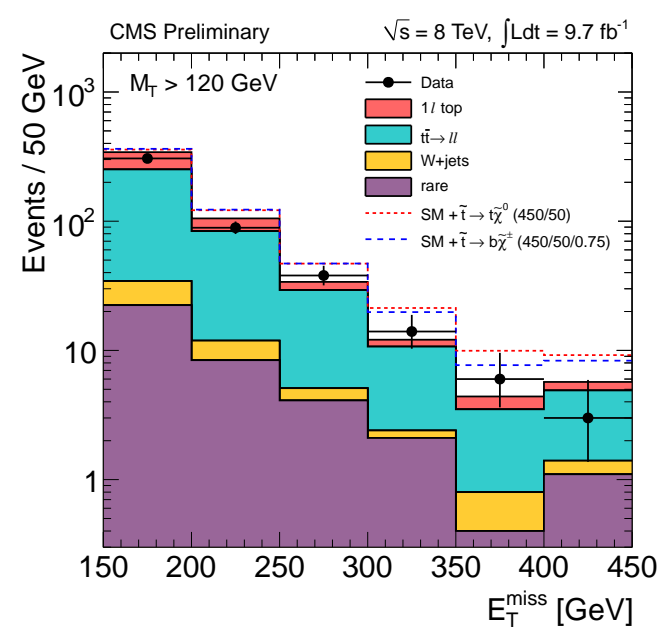

Figure 2: The $\mathrm{E}_{\mathrm{T}}^{\mathrm{miss}}$ distribution in data, compared to the sum of expected backgrounds, for the top squark pair search. Two example signal models with $m_{\tilde{t}}=450 \mathrm{GeV}$ and $m_{\tilde{\chi}_{1}^{0}}=50 \mathrm{GeV}$ are also indicated. For the $\tilde{t} \rightarrow b \tilde{\chi}_{1}^{+}$decay, the chargino mass is set by $m_{\tilde{\chi}_{1}^{ \pm}}=0.75 m_{\tilde{t}}+0.25 m_{\tilde{\chi}_{1}^{0}}$.

per limits on the signal production cross section using, for each model point in the 2-dimensional parameter space, the signal region with the best expected sensitivity. A region of the parameter space is excluded by comparing these cross section upper limits with the theoretical predictions for the signal cross section. Our results probe top squarks with masses up to $430 \mathrm{GeV}$. For comparison, the requirement that SUSY provides a natural solution to the hierarchy problem favors top squarks with masses not exceeding 500-700 GeV [1]. We also interpret our results in the $\tilde{t} \rightarrow b \tilde{\chi}_{1}^{+} \rightarrow b W \tilde{\chi}_{1}^{0}$ scenario depicted in Fig. 1(b), probing top squarks with masses up to $420 \mathrm{GeV}$ [3].

\section{Search in the Same-sign Dilepton Final State}

This section presents a search in the same-sign (SS) dilepton final state, based on $10.5 \mathrm{fb}^{-1}$. A wide variety of new physics scenarios may produce events with SS leptons, which provide a very clean final state due to low SM

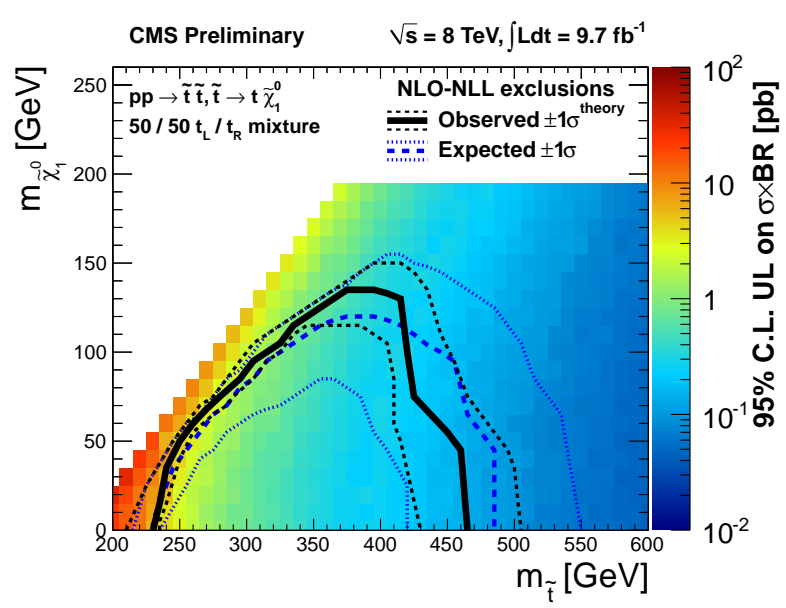

Figure 3: Interpretation of the results of the top squark pair search in the $\tilde{t} \rightarrow t \tilde{\chi}_{1}^{0}$ scenario of Fig. 1(a). The color scale indicates the cross section upper limits at $95 \%$ confidence level. The solid black contour and dashed black contours indicate the observed excluded region and variation in this excluded region due to the $\pm 1 \sigma$ uncertainties in the theoretical prediction of the signal cross section. The dashed blue and dotted blue contours indicate the median and $\pm 1 \sigma$ expected excluded regions.

background expectations. In particular, this final state is sensitive to direct pair production of bottom squarks with $\tilde{b} \rightarrow t \tilde{\chi}_{1}^{-} \rightarrow t W \tilde{\chi}_{1}^{0}$ depicted in Fig. 1(d), as well as to gluino-mediated production of top and bottom squarks.

We select events with two leptons (e or $\mu$ ) with $p_{T}>20$ $\mathrm{GeV}$ and dilepton invariant mass $m_{\ell \ell}>8 \mathrm{GeV}$. We reject events with a third lepton with $p_{T}>10 \mathrm{GeV}$ that forms an opposite-sign same-flavor pair with $76<m_{\ell \ell}<106 \mathrm{GeV}$ with either selected lepton, to suppress the background from $\mathrm{WZ}$ and $\mathrm{ZZ}$. We require the presence of at least two jets with $p_{T}>40 \mathrm{GeV}$.

This analysis is an extension of a previous search in the same-sign dilepton final state [8]. In that analysis, the background is dominated by $t \bar{t} \rightarrow \ell+$ jets where one lepton is from the $\mathrm{W}$ decay and the other lepton is produced in the decay of one of the b-jets. In this analysis we require the presence of at least two b-tagged jets (using CSVM). 


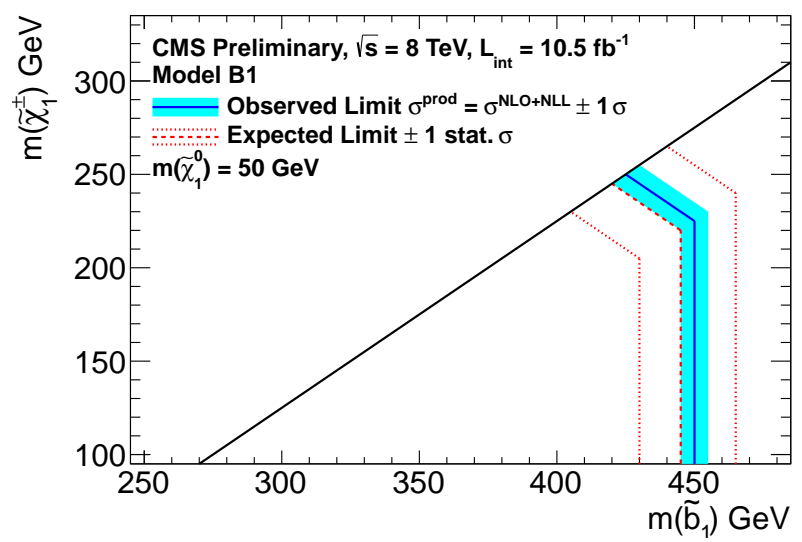

(a)

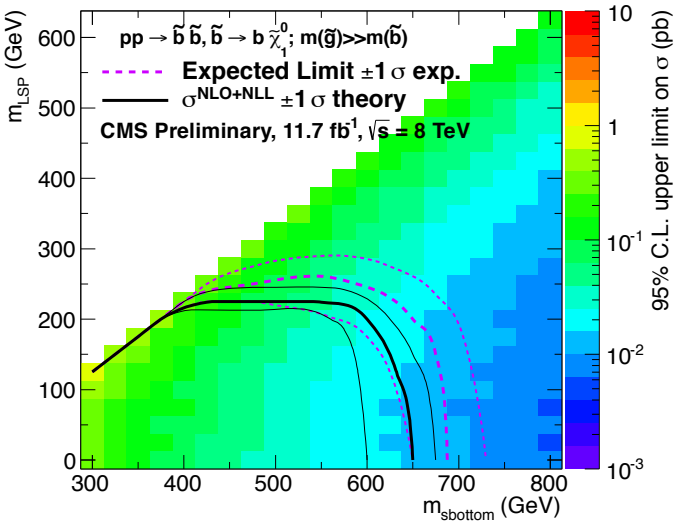

(b)

Figure 4: Interpretation of the results of the search in (a) the same-sign dilepton final state for bottom squark pair production with $\tilde{b} \rightarrow t \tilde{\chi}_{1}^{-}$depicted in Fig. 1(d), and (b) the all-hadronic final state for bottom squark pair production with $\tilde{b} \rightarrow b \tilde{\chi}_{1}^{0}$ depicted in Fig. 1(c).

The requirement that both $b$-jets are identified and wellseparated from the selected leptons reduces the $t \bar{t} \rightarrow \ell+$ jets background by an order of magnitude.

There are three sources of SM background passing the above preselection. The first background source is referred to as "fake leptons" and includes leptons from heavyflavor decay, misidentified hadrons, muons from meson decay in flight, or electrons from unidentified photon conversions. This background is estimated from a sample of events with at least one lepton that passes a loose selection but fails the full analysis identification and isolation requirements. This sample is weighted by the probability for a fake lepton satisfying the loose selection to also pass the analysis selection, which is determined based on studies of fake leptons in jet events. The second background, estimated from MC, consists of rare SM processes and is dominated by $t \bar{t} \mathrm{~W}$ and $t \bar{t} \mathrm{Z}$. The systematic uncertainty on both the fake lepton and rare backgrounds is $50 \%$. A third, small background contribution is from "charge flips" and consists of events with opposite-sign (OS) leptons where one of the leptons is an electron whose charge is misreconstructed. This background is estimated from an OS dilepton data sample, weighted by the electron charge misreconstruction probability, which is extracted from studies of $\mathrm{Z} \rightarrow$ ee events in data and MC.

Several signal regions are defined by placing additional requirements on the jet multiplicity, b-tagged jet multiplicity, $\mathrm{E}_{\mathrm{T}}^{\mathrm{miss}}$, and $H_{T}$, defined as the scalar sum of the transverse momenta of selected jets. The observed data yields in all signal regions are in good agreement with the SM background expectations; see Ref. [4] for the full quantitative results. A signal region that is sensitive to bottom squark pair production with $\tilde{b} \rightarrow t \tilde{\chi}_{1}^{-}$depicted in Fig. 1(d) has at least four jets, $E_{\mathrm{T}}^{\text {miss }}>120 \mathrm{GeV}$ and $H_{T}>200 \mathrm{GeV}$. In this region we observe 1 event in data and predict $2.22 \pm 0.96$ events. The results in all signal regions are used to exclude a region of the model parame- ter space in Fig. 4(a), which demonstrates that our search probes bottom squarks with masses up to $450 \mathrm{GeV}$. Naturalness considerations favor a bottom squark with mass not exceeding 500-700 GeV, similar to the constraint on the top squark mass. Several additional interpretations for models with gluino-mediated top and bottom squark production are presented in Ref. [4].

\section{Search in the All-Hadronic Final State}

The production of bottom squark pairs, followed by the decay $\tilde{b} \rightarrow b \tilde{\chi}_{1}^{0}$, leads to events with two b-jets and $\mathrm{E}_{\mathrm{T}}^{\mathrm{miss}}$. In this section we report the results from a search with 11.7 $\mathrm{fb}^{-1}$ in the all-hadronic final state using the $\alpha_{T}$ variable.

We count jets with $p_{T}>50 \mathrm{GeV}$. The leading (highest $\left.p_{T}\right)$ jet is required to be in the tracker acceptance defined by $|\eta|<2.5$, and the leading two jets must satisfy $p_{T}>$ $100 \mathrm{GeV}$. Events with isolated electrons or muons with $p_{T}$ $>10 \mathrm{GeV}$ are vetoed, in order to suppress backgrounds with neutrinos from the decays of $\mathrm{W}$ bosons. Events with an isolated photon with $p_{T}>25 \mathrm{GeV}$ are vetoed. The remaining events are categorized based on the jet multiplicity, the number of b-tagged jets (using CSVM) and the event $H_{T}$, which is required to satisfy $H_{T}>275 \mathrm{GeV}$.

The background satisfying the above preselection is dominated by QCD multijet production with fake $\mathrm{E}_{\mathrm{T}}^{\mathrm{miss}}$ from mismeasurement effects. To suppress this background, we require the events to have large $\alpha_{T}$. For dijet events this quantity is defined as $\alpha_{T} \equiv E_{T}^{j_{2}} / M_{T}$, where $E_{T}^{j_{2}}$ is the $E_{T}$ of the second leading jet and $M_{T}$ is the transverse mass of the dijet system. For events with perfectly measured jets, the reconstructed $p_{T}$ values of the two jets are equal, leading to $\alpha_{T}=0.5$. The key feature of the $\alpha_{T}$ variable is that mismeasurement effects tend to decrease the value of $\alpha_{T}$, resulting in an endpoint at $\alpha_{T} \approx 0.5$ for the QCD multijet background, which is evident in Fig. 5(a). For events with three of more jets, an equivalent dijet system is formed by clustering the jets into two pseudo-jets. 


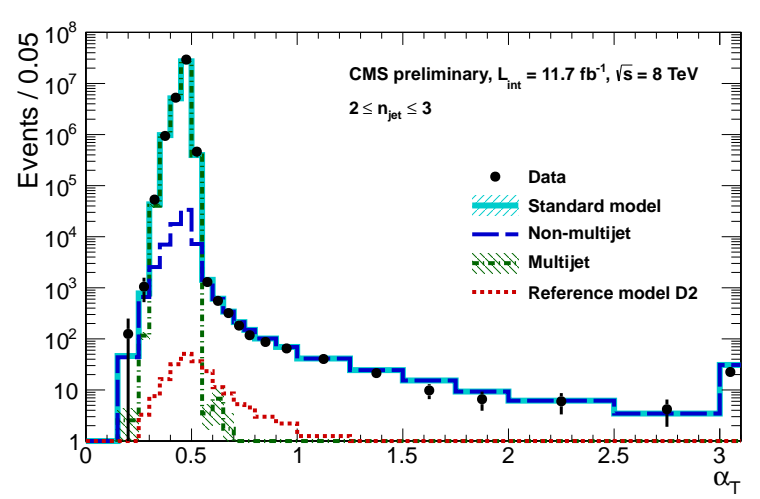

(a)

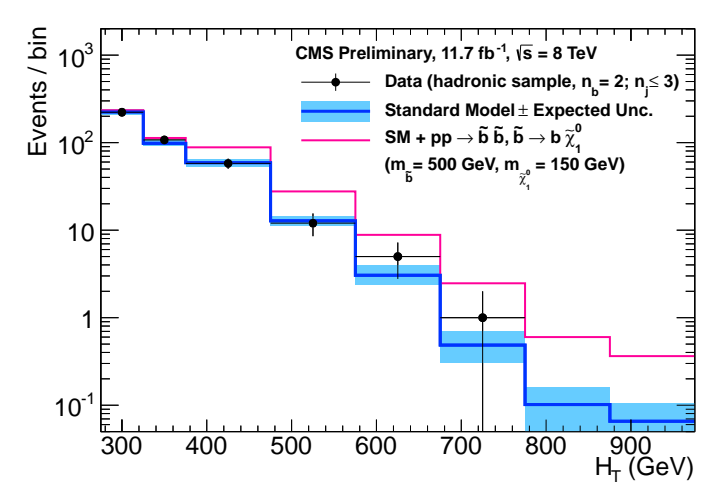

(b)

Figure 5: Distributions of $\alpha_{T}$ (left) and $H_{T}$ (right) in data, compared to the SM background expectations. An example signal scenario of bottom squark pair production with $\tilde{b} \rightarrow b \tilde{\chi}_{1}^{0}$ is overlaid.

In our search we strongly suppress the QCD multijet background with the requirement $\alpha_{T}>0.55$.

The background after the $\alpha_{T}$ requirement is dominated by processes with genuine $\mathrm{E}_{\mathrm{T}}^{\text {miss }}$, including $t \bar{t} \rightarrow \ell+$ jets and $\mathrm{W}+$ jets with a lepton and neutrino from $\mathrm{W}$ decay, where the lepton is either not reconstructed or is a hadronically decaying $\tau$ lepton. These backgrounds are estimated using a $\mu+$ jets data control sample. The additional background from $\mathrm{Z}(v v)+$ jets is estimated using two data control samples of $\mathrm{Z}(\ell \ell)+$ jets and $\gamma+$ jets events. To estimate these backgrounds, the observed yields in the data control samples are extrapolated to the signal region using translation factors derived from MC. The dominant systematic uncertainties in the background prediction stem from the uncertainties in the MC translation factors, which are assessed by performing several closure tests in data. In these tests, the observed yields in one data control region are used to predict the yields in another data control region.

Events are categorized based on the $H_{T}$, jet multiplicity, and b-tagged jet multiplicity. For the bottom squark scenario described above, the most sensitive category is events with either two or three jets and exactly two btagged jets. The $H_{T}$ distribution for these events is indicated in Fig. 5(b), which demonstrates good agreement between the data and the expected background. No evidence for an excess of events is observed.

The results are interpreted using the model of bottom squark pair production with $\tilde{b} \rightarrow b \tilde{\chi}_{1}^{0}$, in Fig. 4(b). These results probe bottom squarks with masses up to approximately $600 \mathrm{GeV}$. Additional interpretations in models with gluino-mediated top and bottom squark pair production are presented in Ref. [5].

\section{Summary}

Supersymmetry may provide a natural solution to the hierarchy problem if it introduces top and bottom squarks with masses not larger than several hundred GeV. In this note we present the results of three searches for the direct pair production of top and bottom squarks. No evidence for event yields beyond the SM expectations is observed. The results are used to place stringent constraints on the masses of top and bottom squarks, in the mass range relevant for the natural supersymmetric solution to the hierarchy problem.

\section{References}

[1] M. Papucci, J. T. Ruderman, and A. Weiler, arXiv:1110.6926 (2011).

[2] CMS Collaboration, JINST 3, S08004 (2008).

[3] CMS Collaboration, CMS-PAS-SUS-12-023 (2012).

[4] CMS Collaboration, arXiv:1212.6194 [hep-ex] (2012).

[5] CMS Collaboration, CMS-PAS-SUS-12-028 (2012).

[6] CMS Collaboration, arXiv:1211.4462 [hep-ex] (2012).

[7] ATLAS Collaboration, Phys. Rev. Lett. 109, 211803 (2012).

[8] CMS Collaboration, Phys. Rev. Lett. 109, 071803 (2012). 